\title{
Abnormal expression of miR-135a in patients with depression and its possible involvement in the pathogenesis of the condition
}

\author{
YINXIA DING ${ }^{1}$, MING ZHONG $^{1}$, BINGJIE QIU ${ }^{1}$, CHUANPENG LIU ${ }^{2}$, JINFENG WANG $^{3}$ and JIE LIANG ${ }^{4}$ \\ ${ }^{1}$ Department of Psychiatry, Binzhou Youfu Hospital; ${ }^{2}$ Department of Psychiatry, \\ Binzhou People's Hospital; ${ }^{3}$ Binzhou Family Planning Association, Binzhou, Shandong 256600; \\ ${ }^{4}$ Ministry of Public Infrastructure, Zhaoqing Medical College, Zhaoqing, Guangdong 526020, P.R. China
}

Received May 18, 2020; Accepted October 9, 2020

DOI: $10.3892 / \mathrm{etm} .2021 .10158$

\begin{abstract}
At present, due to the increasing pressures on society and the stress of everyday living, the number of individuals suffering from depression has increased. Therefore, the treatment of depression has also received increasing attention. MicroRNA (miRNA/miR)-135a is a well-studied miRNA. It has been reported that miR-135a is significantly downregulated in patients with depression and may be a potential marker for the diagnosis of the condition. However, the specific mechanisms of action of miR-135a in patients with depression remain unclear. In the present study, it was found that miR-135a was downregulated in patients with depression, and in a mouse model of depression. The effects of miR-135a on depression-related symptoms in mice were then explored. In the mice with chronic unpredictable mild stress (CUMS) that were treated with miR-135a for 3 weeks, a significantly reduced level of weight gain was observed in comparison with the control group. In addition, treatment with miR-135a mimic significantly increased sucrose preference in the sucrose preference test in the mice, and reduced the immobility time in the forced swimming test and tail suspension test. Treatment with miR-135a mimic also inhibited CUMS-induced hippocampal cell apoptosis. Furthermore, treatment with miR-135a mimic and fluoxetine significantly reduced the CUMS-induced increase in the expression levels of inflammatory factors (IL-1 $\beta$, IL-6 and TNF- $\alpha$ ) in the hippocampus of the mice. Subsequently, reverse transcription-quantitative polymerase chain reaction and western blot analysis revealed that treatment with miR-135a mimic significantly inhibited the expression of Toll-like receptor 4 in the mouse hippocampus. In conclusion, the
\end{abstract}

Correspondence to: Mrs. Jie Liang, Ministry of Public Infrastructure, Zhaoqing Medical College, E4-2106 Luhe Road, Duanzhou, Zhaoqing, Guangdong 526020, P.R. China

E-mail:kj876767@163.com

Key words: microRNA-135a, depression, chronic unpredictable mild stress, Toll-like receptor 4 findings of the present study indicate that miR-135a may be a novel potential target for the treatment of depression.

\section{Introduction}

With the increasing amount of pressure on society and the stress of everyday living, the number of individuals suffering from depression is increasing (1). At present, depression is a common disease which is recognized by the World Health Organization and it is one of the main causes of psychiatric disability worldwide (2). Establishing trust and rapport is the primary intervention strategy for depression, followed by specific interventions according to each diagnosis and the individualized treatment care plan $(3,4)$. Researchers have also highlighted the need for nursing training in order for nurses to perform initial assessment procedures and acquire appropriate evidence-based intervention skills and techniques (3). It has been reported that hippocampal volume and nerve density are markedly reduced in patients with depression through pathophysiological studies (4-6). In addition, a number of studies have demonstrated that the pathogenesis of depression is affected by several factors, such as neural and structural plasticity, neurotransmitter systems, and epigenetic and genetic susceptibility (7-11). Therefore, the study of depression has generally attracted the attention of researchers $(12,13)$.

MicroRNAs (miRNAs/miRs) are a group of endogenous small and non-coding RNAs that regulate gene expression by partially binding to the 3'-untranslated region (3'-UTR) of targeted mRNAs (14-16). Studies have indicated that miRNAs are involved in a number of cellular biological processes in normal physiology and pathogenesis, such as differentiation, cell growth, apoptosis and inflammation (17). Previous research has indicated that miRNAs are highly expressed in the central nervous system and play a crucial role in brain functions, such as neurogenesis, neuronal metabolism, proliferation and apoptosis $(18,19)$.

miR-135a has been found to be upregulated in hepatoma cells, and to facilitate cell proliferation, migration and invasion by targeting FOXO1 and TGFB1 $(20,21)$. Moreover, miR-135a has been found to promote cell proliferation by targeting FOXO1 in malignant melanoma (22). In addition, a previous study demonstrated that miR-135a is related to prostate cancer (23). It has been reported that miR-135a is 
downregulated in the serum of patients with depression and may serve as a potential marker for the diagnosis of depression (24). In addition, Gheysarzadeh et al (24) demonstrated that miR-16 and miR-1202 expression was decreased in the serum of patients with depression. However, the mechanism of action of miR-135a in the development of depression remains unclear.

Currently, chronic unpredictable mild stress (CUMS)induced mouse model of depression has been widely used to investigate depression in vivo (25-27). Therefore, the aim of the present study was to explore the role of miR-135a in CUMS-induced depression and to analyze its molecular mechanisms of action.

\section{Materials and methods}

Clinical samples. In the present study, peripheral blood was obtained from 50 patients with depression (male, 22; female, 28; age range, 37-53 years) and 50 healthy volunteers (male, 24; female, 26; age range, 38-51 years) between January 2018 and December 2019 at Binzhou Youfu Hospital (Binzhou, Shandong, China). Exclusion criteria were a history of bipolar or any psychotic disorder, the use of lithium or an antipsychotic within the prior 2 weeks; substance-use disorder within 3 months; pregnancy or lactation. The healthy controls had no lifetime history of any mental disorder. All patients signed informed consent forms. The present study was approved by the Ethics Committee of Binzhou Youfu Hospital.

Animals and experimental design. A total of 75 male C57BL/6 mice (age, 8-10 weeks; weight, 18-22 g) were purchased from the Nanjing University Animal Research Center. The mice were housed in a standard environment (temperature, $22 \pm 2^{\circ} \mathrm{C}$; humidity, $55 \pm 5 \%$; light/dark cycle, $12 \mathrm{~h}$ ) with free access to food and water. The mice were randomly divided into five groups ( $\mathrm{n}=15$ per group) as follows: i) Unstressed control; ii) CUMS; iii) CUMS + $0.5 \mathrm{nmol}$ mimic control injected intraperitoneally (5'-UUUGUACUACACAAAAGUACUG-3'; Guangzhou RiboBio Co.,Ltd.); iv) CUMS + 0.5 nmol miR-135a mimic injected intraperitoneally (5'-UAUGGCUUUUUA UUCCUAUGUGA-3'; Guangzhou RiboBio Co., Ltd.); and v) positive control group injected intraperitoneally with $20 \mathrm{mg} / \mathrm{kg} / \mathrm{day}$ fluoxetine (Sigma-Aldrich; Merck KGaA) for 21 days (CUMS + FLU). All animal experiments were performed according to a protocol approved by the Committee on Care and Use of the Laboratory Animal Committee of Binzhou Youfu Hospital.

A total of 3 weeks after mimic control/miR-135a mimic/FLU treatment, the mice were anesthetized with an intraperitoneal injection of pentobarbital (Sigma-Aldrich; Merck KGaA; $50 \mathrm{mg} / \mathrm{kg}$ ) and sacrificed by cervical dislocation. Death was defined as the lack of heartbeat and breathing. The peripheral blood and hippocampus tissue were subsequently harvested following euthanasia as previously described (28).

Mouse model of CUMS. A mouse model of depression was established by inducing CUMS, as previously described (25). In brief, the CUMS procedures included the following various mild stress factors: Continuous night illumination (overnight), cage tilt ( $7 \mathrm{~h})$, water and food deprivation (24 h), swimming in cold water, noise, wet pads, foreign object exposure $(6 \mathrm{~h})$, tail clamp ( $1 \mathrm{~min})$, hanging of the mice on the balance bar with a rope $(10 \mathrm{~min})$, physical restraint for $3 \mathrm{~h}$ and a 5 -min oscillation. The mice were subjected to 2-3 kinds of stimuli each day; however, the same stressor was not applied again within less than 3 consecutive days. The CUMS procedure lasted for 6 weeks, and treatments with $\mathrm{miR}-135 \mathrm{a} \mathrm{mimic} / \mathrm{mimic}$ control/FLU were performed daily from the 4th to the 6th week. The body weights of the mice were monitored from the beginning of the experiment and measured each week.

Sucrose preference test (SPT). The SPT was performed using the method previously described by Iñiguez et al (29). SPT was performed every week. Briefly, the mice were deprived of water and food for $24 \mathrm{~h}$ and then tested for sucrose preference. Each mouse was given free access to two bottles for $12 \mathrm{~h}$ : One bottle contained $1 \%$ sucrose solution $(\mathrm{w} / \mathrm{v})$ and the other bottle contained tap water. To avoid the influence of the positioning, the two bottles were placed opposite each other. After $12 \mathrm{~h}$, the consumed volumes of tap water and sucrose solution were recorded. The sucrose preference was calculated as (sucrose solution intake)/(sucrose solution intake + tap water intake) $\mathrm{x} 100 \%$.

Forced swimming test (FST). After 3 weeks of mimic control/miR-135a mimic/FLU treatment, FST was performed. The FST was carried out in a cylindrical container (height, $65 \mathrm{~cm}$; diameter, $30 \mathrm{~cm}$ ), which was filled to a height of $40 \mathrm{~cm}$ with water (temperature, $22-23^{\circ} \mathrm{C}$ ). The FST lasted for $6 \mathrm{~min}$ and the immobility time (in sec) was recorded during the last $4 \mathrm{~min}$. The immobility time was defined as the time when the mouse remained still and not struggling, using only the basic motion to maintain its head on the water, or touching the bottom for $>1$ sec.

Tail suspension test (TST). After 3 weeks of mimic control/miR-135a mimic/FLU treatment, the TST was performed as previously described (30). Briefly, the tape was placed at a distance of $1 \mathrm{~cm}$ from the extremity of the tail of the mouse to fix the position. The mice were suspended for $6 \mathrm{~min}$ and immobility was recorded during the last $4 \mathrm{~min}$.

Reverse transcription-quantitative polymerase chain reaction $(R T-q P C R)$. Total RNA was extracted from the murine hippocampus tissue or human and murine peripheral blood using TRIzol ${ }^{\circledR}$ reagent (Thermo Fisher Scientific, Inc.) following the manufacturer's protocol. Total RNA concentration was detected using a NanoDrop 2000 spectrophotometer (NanoDrop; Thermo Fisher Scientific, Inc.). Total RNA was stored at $-80^{\circ} \mathrm{C}$ until use. The synthesis of cDNA was performed using the RevertAid ${ }^{\mathrm{TM}}$ First Strand cDNA Synthesis kit (Vazyme Biotech Co., Ltd.). The reaction conditions were as follows: $70^{\circ} \mathrm{C}$ for $5 \mathrm{~min}, 37^{\circ} \mathrm{C}$ for $5 \mathrm{~min}$ and $42^{\circ} \mathrm{C}$ for $60 \mathrm{~min}$. SYBR-Green (Vazyme Biotech Co., Ltd.) qPCR assay was performed to measure the expression level of the target gene. The thermocycling conditions were as follows: Initial denaturation at $95^{\circ} \mathrm{C}$ for $5 \mathrm{~min} ; 40$ cycles of denaturation at $95^{\circ} \mathrm{C}$ for $10 \mathrm{sec}$, annealing at $60^{\circ} \mathrm{C}$ for $20 \mathrm{sec}$ and extension at $72^{\circ} \mathrm{C}$ for $30 \mathrm{sec}$. Relative expression levels were calculated 
using the $2^{-\Delta \Delta \mathrm{Cq}}$ method following normalization with reference to the expression of GAPDH or U6 (31). All experiments were performed in triplicate to ensure minimum deviation. The following primer sequences were used: GAPDH forward, 5'-TTTGGTATCGTGGAAGGACTC-3' and reverse, 5'-GTA GAGGCAGGGATGATGTTCT-3'; U6 forward, 5'-GCTTCG GCAGCACATATACTAAAAT-3' and reverse, 5'-CGCTTC ACGAATTTGCGTGTCAT-3'; Toll-like receptor 4 (TLR4) forward, 5'-CCTGACACCAGGAAGCTTGAA-3' and reverse, 5'-TCTGATCCATGCATTGGTAGGT-3'; miR-135a forward, 5'-ACACTCCAGCTCAGTATGGCTTTTTATTCCTATGT-3' and reverse, 5'-CTCAACTGGTGTCGTGGAGTCGGCAAT TCAG-3'.

Western blot analysis. Protein was extracted using RIPA buffer with $1 \mathrm{mM}$ protease inhibitor PMSF (Beijing Solarbio Science \& Technology Co., Ltd.). A bicinchoninic acid protein assay kit (Sigma-Aldrich; Merck KGaA) was applied to determine protein concentration. Protein (30 mg per lane) was separated on $10 \%$ SDS gels and transferred onto PVDF membranes (EMD Millipore). The membranes were blocked with $5 \%$ skim milk with TBS containing $0.1 \%$ Tween-20 for $1 \mathrm{~h}$ at room temperature and incubated with the following primary antibodies: Anti-Bcl-2 (cat. no. 3498; 1:1,000), anti-Bax (cat. no. $2772 ; 1: 1,000$ ), anti-TLR4 (cat. no. 14358; 1:1,000) and anti-GAPDH (cat. no. 5174; 1:1,000) (all purchased from Cell Signaling Technology, Inc.) at $4^{\circ} \mathrm{C}$ overnight. The membranes were then incubated with horseradish peroxidase-conjugated secondary antibody (goat anti-rabbit; cat. no. 7074; 1:2,000; Cell Signaling Technology, Inc.) for $2 \mathrm{~h}$ at room temperature. Subsequently, the protein bands were detected and visualized by RapidStep ${ }^{\mathrm{TM}}$ ECL Reagent (EMD Millipore). Band densities were quantified using Gel-Pro Analyzer Densitometry software (version 6.3; Media Cybernetics, Inc.).

ELISA. After treatment, mice were sacrificed and hippocampus were immediately dissected and then stored at $-80^{\circ} \mathrm{C}$. After the samples were homogenized and centrifuged at 5,000 x g at $4^{\circ} \mathrm{C}$ for $15 \mathrm{~min}$, the supernatant was collected. ELISA was performed to examine the expression levels of TNF- $\alpha$ (cat. no. PT518), IL-6 (cat. no. PI330) and IL-1 $\beta$ (cat. no. PI305) in the mouse hippocampus using ELISA kits according to the manufacturer's instructions (all manufactured by Beyotime Institute of Biotechnology).

Flow cytometric analysis. Cell apoptosis was analyzed using the Annexin V/PI Apoptosis Detection kit (BD Biosciences). The hippocampus was dissected as aforementioned and dissociated into a single cell suspension by enzymatic degradation using a neural tissue dissociation kit (Miltenyi Biotec, Inc.). The cells were then collected, centrifuged at low temperature and high speed $(1,000 \mathrm{x} \mathrm{g}$ at $4^{\circ} \mathrm{C}$ for $5 \mathrm{~min}$ ), and re-suspended in $100 \mu \mathrm{l} \mathrm{FITC} \mathrm{binding}$ buffer. Subsequently, the buffer was supplemented with $\sim 5 \mu \mathrm{l}$ ready-to-use Annexin V-FITC and $5 \mu \mathrm{l}$ PI. In the dark, the cells were incubated for $30 \mathrm{~min}$ at room temperature. Annexin V-FITC and PI fluorescence were assessed using a BD FACSCalibur flow cytometer (BD Biosciences). Data were analyzed using FlowJo software (version 7.2.4; FlowJo LLC).
Statistical analysis. Data are presented as the mean \pm SD from at least three independent experiments. GraphPad 6.0 software (GraphPad Software, Inc.) was used for statistical analysis and unpaired Student's t-test was performed to determine whether differences between two groups were significant. Differences among multiple groups were analyzed by one-way ANOVA followed by a Tukey's post hoc test. $\mathrm{P}<0.05$ was considered to indicate a statistically significant difference.

\section{Results}

Expression of miR-135a in patients with depression and in the mouse model of depression. To explore the role of miR-135a in patients with depression and in the mouse model of depression, peripheral blood was collected from 50 patients with depression and 50 healthy subjects, and the expression of miR-135a was detected by RT-qPCR. The results of RT-qPCR revealed that compared with the healthy individuals, miR-135a expression was significantly reduced in patients with depression (Fig. 1A). Subsequently, a mouse model of depression was established by inducing CUMS for 6 weeks. Peripheral blood and hippocampal tissue samples were then collected from the mice, and the expression of miR-135a was detected by RT-qPCR. The results revealed that compared with the control group, the expression of miR-135a was decreased in mouse peripheral blood (Fig. 1B) and in the hippocampus (Fig. 1C) in the CUMS group. These results indicated that miR-135a expression was downregulated in patients and mice with depression.

Effect of miR-135a on the body weights of mice and depressive-like behavior. The present study first confirmed that compared with the mimic control group, miR-135a mimic significantly enhanced the level of miR-135a in the hippocampal tissue of mice (Fig. 2A). At the beginning of the experiment, the weights of the mice in the different groups were similar. Following 2 weeks of CUMS, the body weights of the mice in the model group were significantly lower than those in the control group. Treatment with miR-135a mimic and FLU significantly attenuated the CUMS-induced reduction in weight gain (Fig. 2B). The effects of miR-135a on depression-related symptoms in mice were then examined. The SPT was determined every week. And following treatment of the mice subjected to CUMS with miR-135a mimic for 3 weeks, the FST and TST were used to evaluate the anti-depressant effects of miR-135a mimic. Compared with CUMS + mimic control group, treatment with miR-135a mimic and FLU markedly decreased CUMS-induced depression-like behavior at 3 weeks after treatment. Compared with the mice in the CUMS + mimic control group, miR-135a mimic significantly increased SPT (1, 2 and 3 weeks after treatment) in mice and reduced the immobility time in the FST and TST (Fig. 2C-E). These results indicated that miR-135a treatment relieved CUMS-induced depressive-like behavior.

Effect of miR-135a on CUMS-induced hippocampal cell apoptosis. Subsequently, the effects of miR-135a on the apoptosis of neural cells in the mouse hippocampus were examined by flow cytometry. Compared with the control group, hippocampal neuronal apoptosis was significantly increased in the 

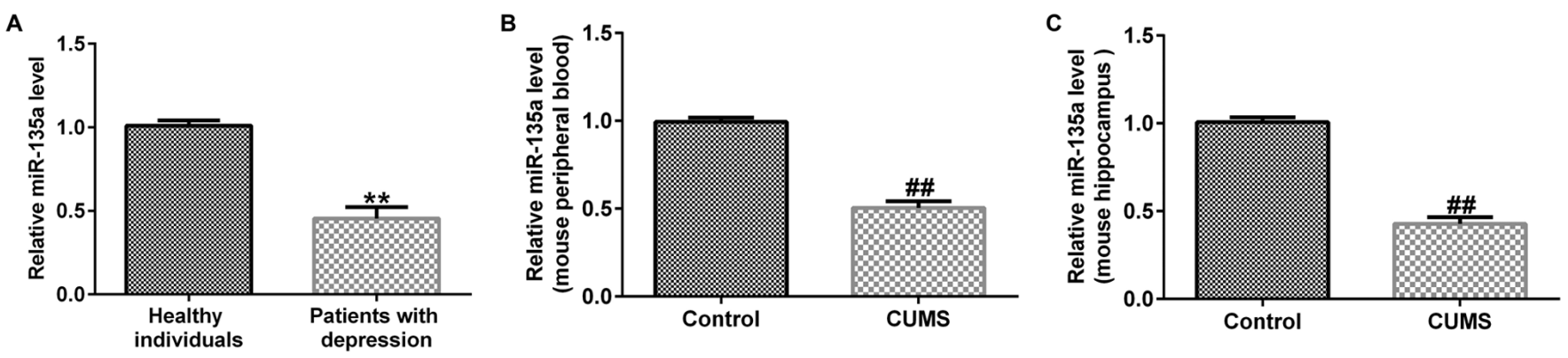

Figure 1. miR-135a expression is decreased in patients and mice with depression. (A) miR-135a expression levels were evaluated by reverse transcription-quantitative polymerase chain reaction in peripheral blood from 50 patients with depression and 50 healthy individuals. Expression of miR-135a in mouse (B) peripheral blood and (C) hippocampus in the control and CUMS groups. Data are presented as the mean \pm SD. ${ }^{* *} \mathrm{P}<0.01$ vs. healthy individuals; ${ }^{\# \#} \mathrm{P}<0.01$ vs. control. CUMS, chronic unpredictable mild stress; miR, microRNA.

A
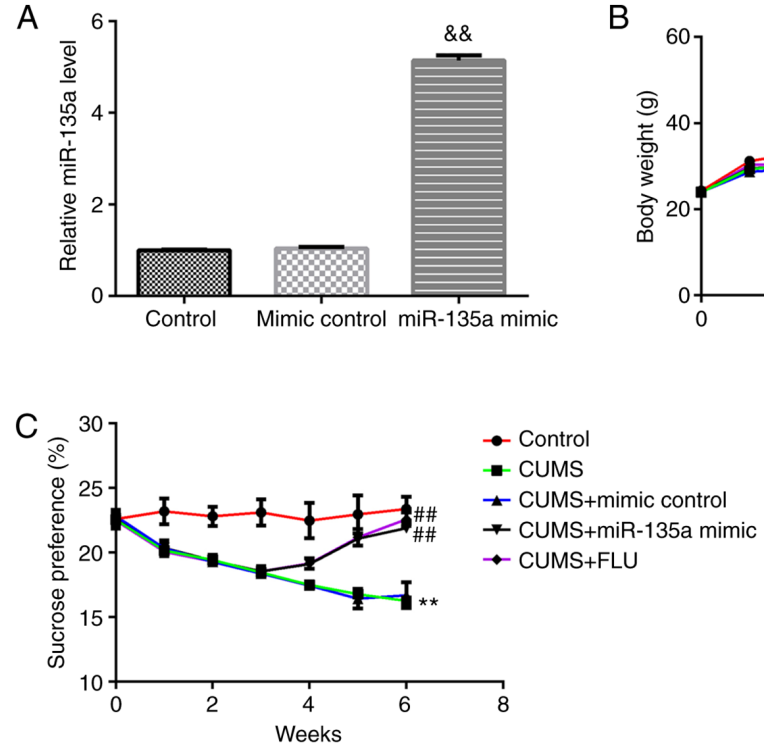

B

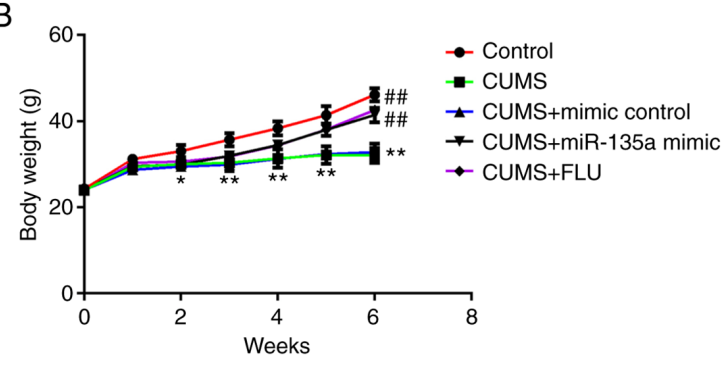

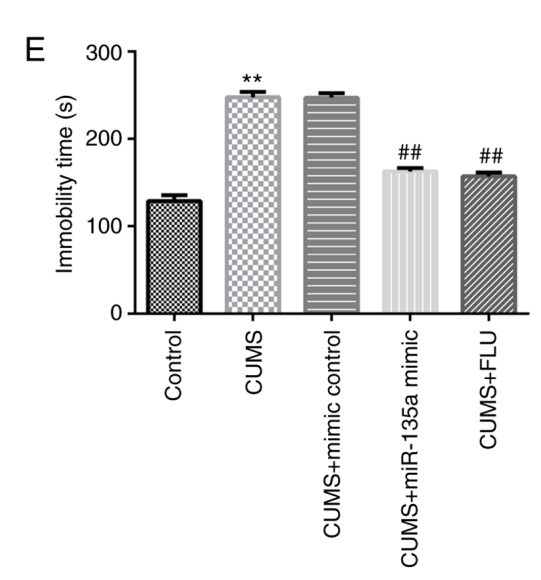

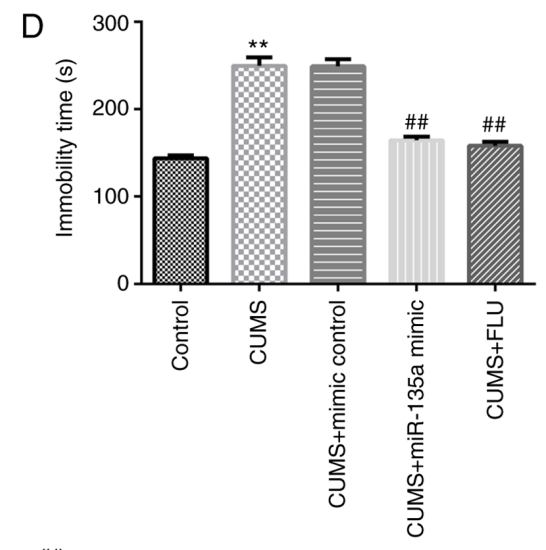

2. miR-135a attenuates CUMS-induced weight loss in mice and symptoms associated with CUMS in mice. (A) miR-135a level in the hippocampal tissue of mice was detected using reverse transcription-quantitative polymerase chain reaction. (B) Body weight of mice. (C) Sucrose preference test results.

(D) Forced swimming test results. (E) Tail suspension test results. Data are presented as the mean $\pm \mathrm{SD}$. ${ }^{\& \&} \mathrm{P}<0.01$ vs. mimic control group; ${ }^{*} \mathrm{P}<0.05$ and ${ }^{* *} \mathrm{P}<0.01$ vs control group; ${ }^{\# \#} \mathrm{P}<0.01$ vs. CUMS + mimic control group. CUMS, chronic unpredictable mild stress; FLU, fluoxetine; miR, microRNA.

CUMS group (Fig. 3A and B). However, the apoptotic rate in the hippocampus was decreased in the miR-135a mimicand FLU-treated group compared with the CUMS group (Fig. 3A and B). In addition, compared with the control group,
Bax protein expression was increased, $\mathrm{Bcl}-2$ protein expression was decreased, and the Bax/Bcl-2 ratio was increased in the CUMS group (Fig. 3C and D). However, miR-135a mimic and FLU treatment significantly reduced the expression of 

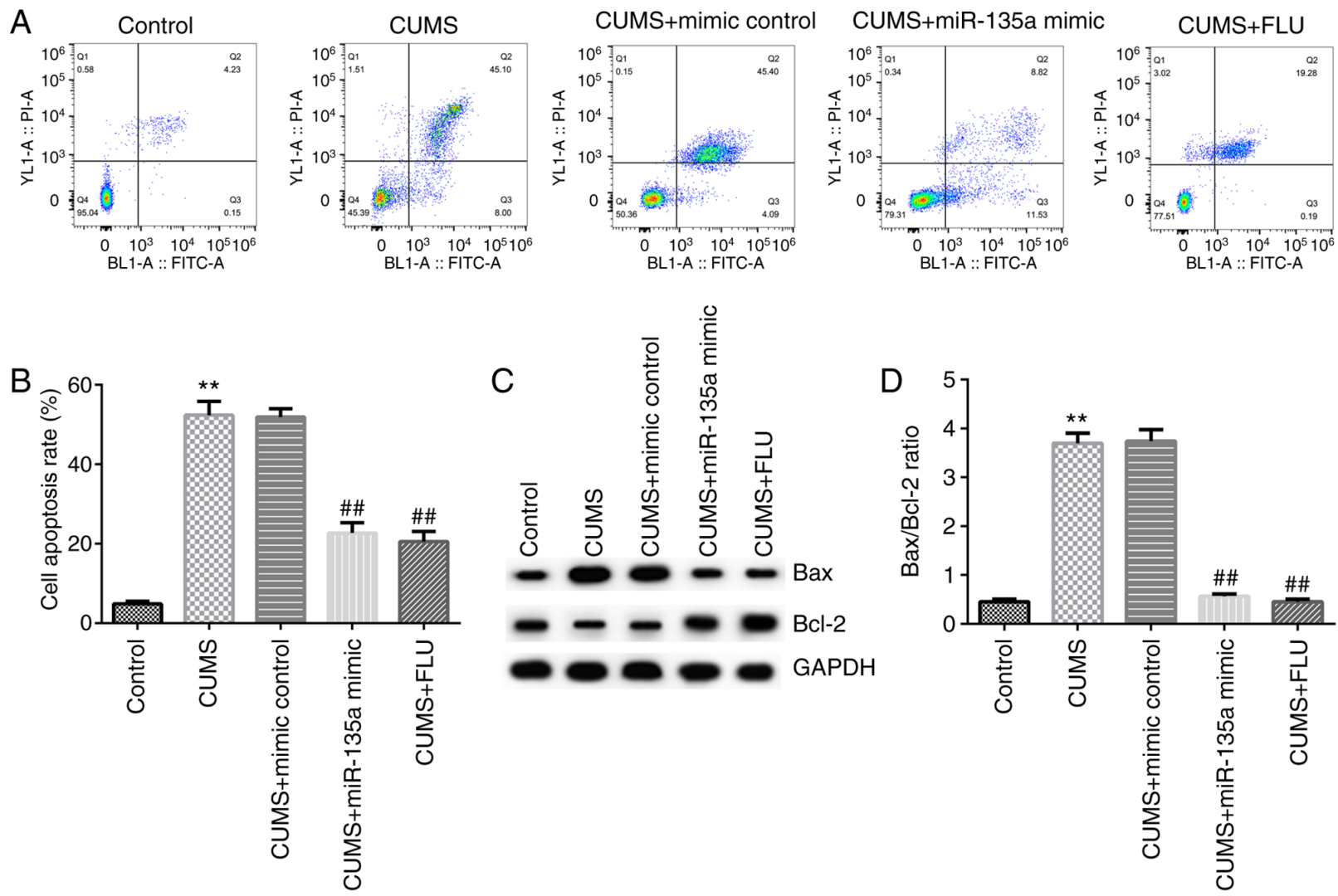

Figure 3. miR-135a inhibits CUMS-induced hippocampal cell apoptosis. (A and B) Flow cytometric analysis of cell apoptosis in mice in the different groups (C) Western blot analysis of the protein expression of Bax and Bcl-2. (D) Ratio of Bax/Bcl-2. Data are presented as the mean \pm SD. . ${ }^{* *}<0.01$ vs. control group; ${ }^{\#} \mathrm{P}<0.01$ vs. CUMS + mimic control group. CUMS, chronic unpredictable mild stress; FLU, fluoxetine; miR, microRNA.

A

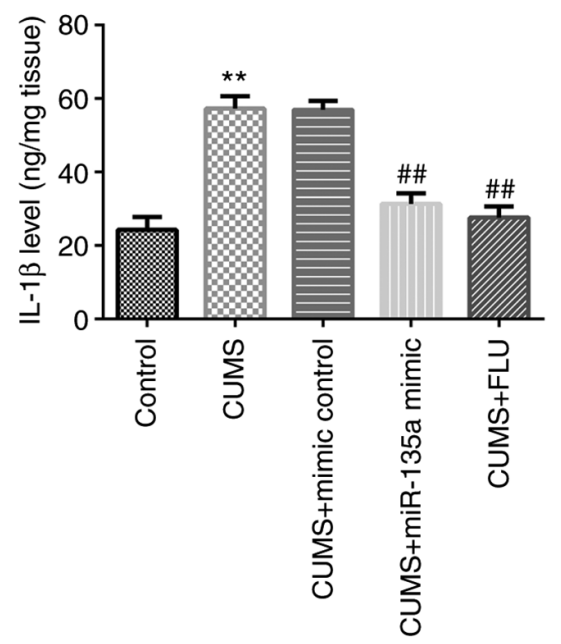

B

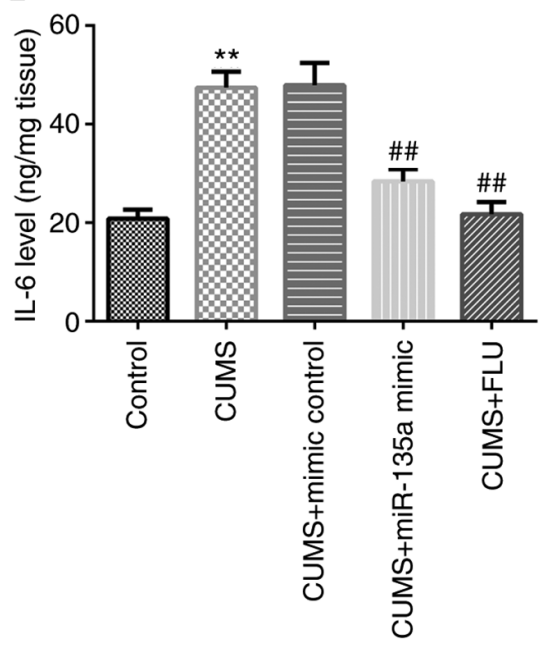

C

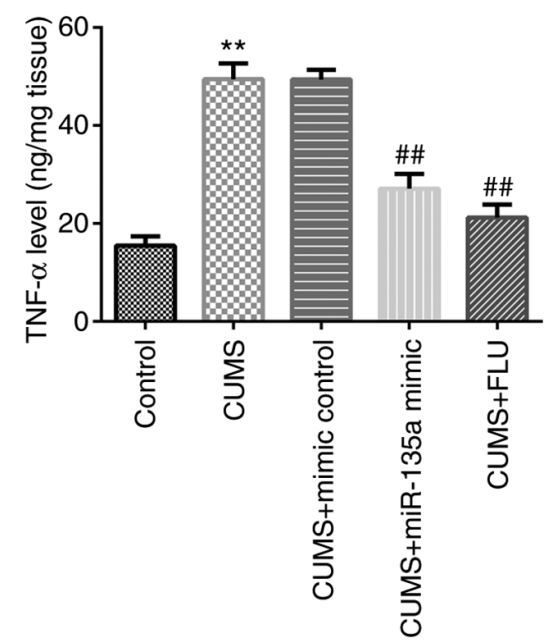

Figure 4. miR-135a attenuates the CUMS-induced hippocampal inflammatory response. ELISA of the protein levels of (A) IL-1 $\beta$, (B) IL-6 and (C) TNF- $\alpha$ in the hippocampus of mice. Data are presented as the mean $\pm \mathrm{SD}$. ${ }^{* *} \mathrm{P}<0.01$ vs. control group; ${ }^{\# \#} \mathrm{P}<0.01 \mathrm{vs}$. CUMS + mimic control group. CUMS, chronic unpredictable mild stress; FLU, fluoxetine; miR, microRNA.

Bax, increased Bcl-2 expression and decreased the Bax/Bcl-2 ratio (Fig. $3 \mathrm{C}$ and $\mathrm{D}$ ). Taken together, these results indicate that miR-135a inhibited CUMS-induced hippocampal cell apoptosis.

Effects of miR-135a on the CUMS-induced hippocampal inflammatory response. Subsequently, to examine the effects of miR-135a treatment on neuroinflammation, ELISA was performed to detect the levels of inflammatory factors in the hippocampus. The results revealed that the levels of IL-1 $\beta$, IL- 6 and TNF- $\alpha$ were increased in the CUMS group compared with the control group (Fig. 4A-C). However, miR-135a mimic and FLU treatment significantly attenuated the CUMS-induced increase in the levels of inflammatory factors in the hippocampus of mice (Fig. 4A-C). 
A

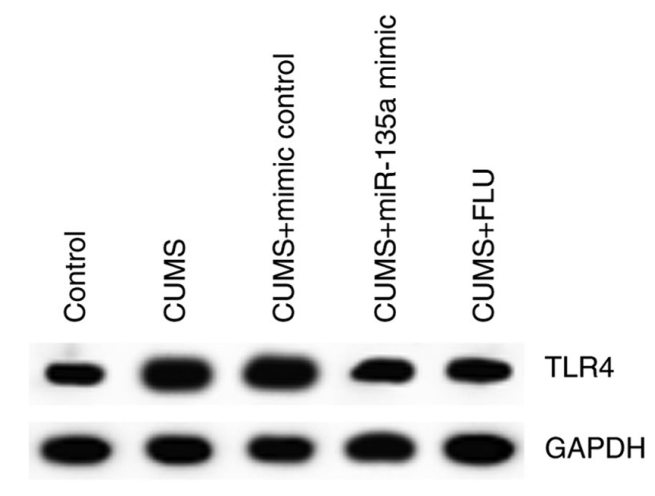

$\mathrm{B}$

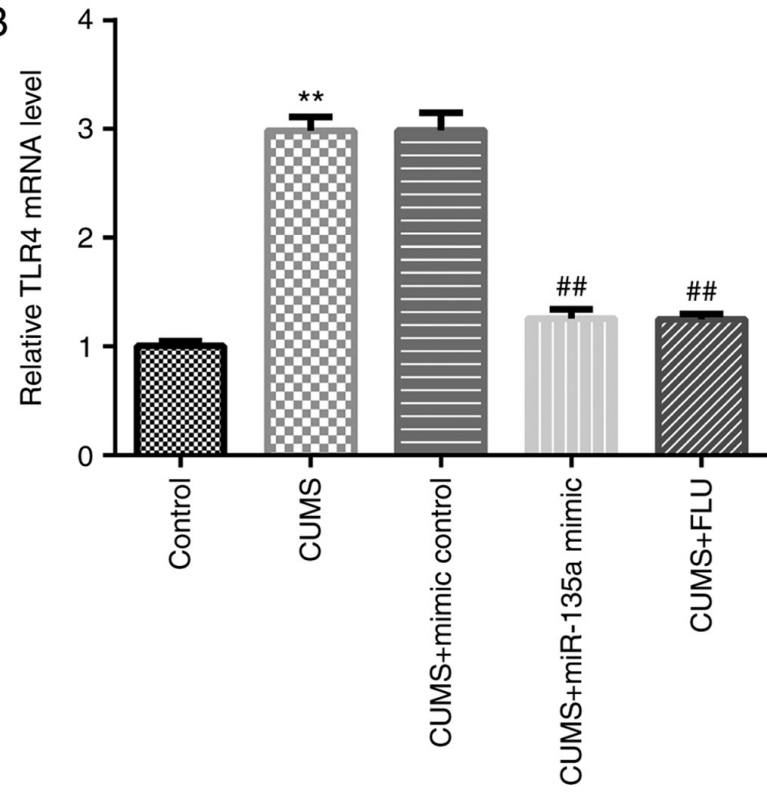

Figure 5. Expression of TLR4 in mice with depression. (A) Western blot analysis of TLR4 protein expression. (B) Reverse transcription-quantitative polymerase chain reaction assay of TLR4 mRNA expression. Data are presented as the mean $\pm \mathrm{SD}$. ${ }^{* *} \mathrm{P}<0.01$ vs. control group; ${ }^{\# \#} \mathrm{P}<0.01 \mathrm{vs}$. CUMS + mimic control group. CUMS, chronic unpredictable mild stress; FLU, fluoxetine; miR, microRNA; TLR4, Toll-like receptor 4.

Therefore, miR-135a decreased the CUMS-induced hippocampal inflammatory response.

miR-135a may protect against depression in mice by regulating TLR4 expression. A previous study demonstrated that TLR4 was a target gene of miR-135a (32). Therefore, in order to elucidate whether miR-135a affects depression by regulating the expression of TLR4, the expression of TLR4 was examined in the mice following treatment. After 3 weeks, the expression of TLR4 was detected in the hippocampus of the mice in each group by RT-qPCR and western blot analysis. The results revealed that compared with the control group, the mRNA expression of TLR4 was significantly increased in the CUMS group, and this increase was significantly inhibited by miR-135a mimic (Fig. 5A and B).

\section{Discussion}

Depression is a common illness that severely influences the quality of life. In recent years, an increasing number of studies have focused on the exploration of the role of miRNA in the pathological mechanism of depression (33-35). It has previously been reported that miR-135a exerts an inhibitory effect in prostate cancer and lung cancer $(36,37)$. Furthermore, miR-135a has been reported to function as an oncogene in gastric and colorectal cancer $(38,39)$. In addition, Shi et al (40) indicated that miR-135a expression was downregulated in glioma tissues and cells. However, to the best of our knowledge, there are few studies available on the expression and mechanisms of action of miR-135a in patients with depression. Gheysarzadeh et al (24) demonstrated that miR-135a expression was decreased in patients with depression. CUMS-induced animal models are currently considered as one of the most appropriate animal models for studying depression $(25-28,41)$. CUMS exposure can induce depression-like behavior in animals, such as behavioral despair, a lack of pleasure and reduced exercise tolerance (42). In the present study, it was found that the expression of miR-135a was downregulated in patients with depression and in a mouse model of CUMS-induced depression.

Wan et al (42) demonstrated that miR-29b-3p overexpression improved depressive-like behaviors in rats with CUMS. In the current study, the effects of miR-135a on depression-related symptoms in mice with depression were examined. It was found that miR-135a attenuated CUMS-induced in mice. In addition, miR-135a mimic significantly increased SPT in mice and shortened the immobility time in the FST and TST. The results of the present study also demonstrated that miR-135a inhibited CUMS-induced hippocampal cell apoptosis. There is increasing evidence to indicate that inflammation is the primary pathological mechanism of depression (43). Psychological and physical stressors can stimulate immune and inflammatory processes (44). Previous studies have demonstrated that the increase in the levels of pro-inflammatory cytokines (IL-1 $\beta$, IL-6 and TNF- $\alpha$ ) in certain regions of the brain, such as the hippocampus of depressed mice, is associated with the pathophysiology of depression $(45,46)$. In the present study, miR-135a attenuated the CUMS-induced hippocampal inflammatory response and reduced the CUMS-induced increase in the levels of inflammatory factors, including IL-1 $\beta$, IL-6 and TNF- $\alpha$ in the hippocampus of mice.

All the aforementioned results indicate that miR-135a has a certain alleviating effect on depression. Du and Lu (47) demonstrated that TLR4 was the direct target gene of miR-135a, and miR-135a suppressed oxidative stress and vascular inflammatory events through TLR4 in atherogenesis. TLR4 plays an important role in the development process of atherosclerosis (48) and acts as a potential target of miR-590 (49). The findings of the present study demonstrated that miR-135a may protect mice against depression by regulating TLR4 expression. 
In conclusion, the present study demonstrated that miR-135a regulated the apoptosis and inflammatory response in mouse hippocampal neurons by regulating the expression of TLR4, thereby alleviating the depressive behavior of mice and playing a protective role in depression.

\section{Acknowledgements}

Not applicable.

\section{Funding}

No funding was received.

\section{Availability of data and materials}

The datasets used and/or analyzed during the present study are available from the corresponding author on reasonable request.

\section{Authors' contributions}

YXD conceived and designed the current study, acquired, analyzed and interpreted the data, and prepared the manuscript. MZ, BJQ, CPL and JFW contributed to the acquisition and analysis of the data. JL contributed to acquisition and analysis of the data and prepared the manuscript. YXD and JL confirm the authenticity of all the raw data. All authors read and approved the final manuscript.

\section{Ethics approval and consent to participate}

All patients signed informed consent forms. The present study was approved by the Ethics Committee of Binzhou Youfu Hospital (Binzhou, China). All animal experiments were performed according to a protocol approved by the Committee on Care and Use of the Laboratory Animal Committee of Binzhou Youfu Hospital.

\section{Patient consent for publication}

All patients consented to the publication of their data.

\section{Competing interests}

The authors declare that they have no competing interests.

\section{References}

1. Bortolato B, Carvalho AF, Soczynska JK, Perini GI and McIntyre RS: The involvement of TNF- $\alpha$ in cognitive dysfunction associated with major depressive disorder: An opportunity for domain specific treatments. Curr Neuropharmacol 13: 558-576, 2015.

2. Belmaker RH and Agam G: Major depressive disorder. N Engl J Med 358: 55-68, 2008.

3. Prokofieva M, Koukia E and Dikeos D: Mental health nursing in Greece: Nursing diagnoses and interventions in major depression. Issues Ment Health Nurs 37: 556-562, 2016.

4. Chuang YH and Kuo LM: Nurses' confidence in providing and managing care for older persons with depressive symptoms or depression in long-term care facilities: A national survey. Int $\mathbf{J}$ Ment Health Nurs 27: 1767-1775, 2018.
5. Xie X, Shi Y and Zhang J: Structural network connectivity impairment and depressive symptoms in cerebral small vessel disease. J Affect Disord 220: 8-14, 2017.

6. Suzuki H, Matsumoto Y, Ota H, Sugimura K, Takahashi J, Ito K, Miyata S, Furukawa K, Arai H, Fukumoto Y, et al: Hippocampal blood flow abnormality associated with depressive symptoms and cognitive impairment in patients with chronic heart failure. Circ J 80: 1773-1780, 2016.

7. Nabavi SM, Daglia M, Braidy N and Nabavi SF: Natural products, micronutrients, and nutraceuticals for the treatment of depression: A short review. Nutr Neurosci 20: 180-194, 2017.

8. Weil-Malherbe H: The biochemistry of affective disorders. In: Handbook of Neurochemistry. Springer, Boston, MA, pp371-416, 1972.

9. Lee S, Jeong J, Kwak Y and Park SK: Depression research: Where are we now? Mol Brain 3: 8-0, 2010.

10. Ebmeier KP, Donaghey C and Steele JD: Recent developments and current controversies in depression. Lancet 367: 153-167, 2006.

11. Leistedt SJ and Linkowski P: Brain, networks, depression, and more. Eur Neuropsychopharmacol 23: 55-62, 2013.

12. Kessler RC: The costs of depression. Psychiatr Clin North Am 35: 1-14, 2012.

13. Ota KT and Duman RS: Environmental and pharmacological modulations of cellular plasticity: Role in the pathophysiology and treatment of depression. Neurobiol Dis 57: 28-37, 2013.

14. Bartel DP: MicroRNAs: Genomics, biogenesis, mechanism, and function. Cell 116: 281-297, 2004.

15. Calin GA, Sevignani C, Dumitru CD, Hyslop T, Noch E, Yendamuri S, Shimizu M, Rattan S, Bullrich F, Negrini M, et al: Human microRNA genes are frequently located at fragile sites and genomic regions involved in cancers. Proc Natl Acad Sci USA 101: 2999-3004, 2004.

16. Krol J, Loedige I and Filipowicz W: The widespread regulation of microRNA biogenesis, function and decay. Nat Rev Genet 11: 597-610, 2010.

17. Mendell $\mathbf{J}$ and Olson E. MicroRNAs in stress signaling and human disease. Cell 148: 1172-1187, 2012.

18. Maes OC, Chertkow HM, Wang E and Schipper HM: MicroRNA: Implications for Alzheimer disease and other human CNS disorders. Curr Genomics 10: 154-168, 2009.

19. Kocerha J, Kauppinen S and Wahlestedt C: MicroRNAs in CNS disorders. Neuromolecular Med 11: 162-172, 2009.

20. Zeng YB, Liang XH, Zhang GX, Jiang N, Zhang T, Huang JY, Zhang L and Zeng XC: miRNA-135a promotes hepatocellular carcinoma cell migration and invasion by targeting forkhead box O1. Cancer Cell Int 16: 63, 2016.

21. Yao S, Tian C, Ding Y, Ye Q, Gao Y, Yang N and Li Q: Down-regulation of Krüppel-like factor-4 by microRNA-135a-5p promotes proliferation and metastasis in hepatocellular carcinoma by transforming growth factor- $\beta 1$. Oncotarget 7 : 42566-42578, 2016.

22. Ren JW, Li ZJ and Tu C: miR-135 post-transcriptionally regulates FOXO1 expression and promotes cell proliferation in human malignant melanoma cells. Int J Clin Exp Pathol 8: 6356-6366, 2015.

23. Xu B, Lu X, Zhao Y, Liu C, Huang X, Chen S, Zhu W, Zhang L and Chen M: MicroRNA-135a induces prostate cancer cell apoptosis via inhibition of STAT6. Oncol Lett 17: 1889-1895, 2019.

24. Gheysarzadeh A, Sadeghifard N, Afraidooni L, Pooyan F, Mofid MR, Valadbeigi H, Bakhtiari $\mathrm{H}$ and Keikhavani S: Serum-based microRNA biomarkers for major depression: miR-16, miR-135a, and miR-1202. J Res Med Sci 23: 69, 2018.

25. Deng XY, Li HY, Chen JJ, Li RP, Qu R, Fu Q and Ma SP: Thymol produces an antidepressant-like effect in a chronic unpredictable mild stress model of depression in mice. Behav Brain Res 291: 12-19, 2015.

26. Chen YP, Wang C and Xu JP: Chronic unpredictable mild stress induced depression-like behaviours and glutamate-glutamine cycling dysfunctions in both blood and brain of mice. Pharm Biol 57: 280-286, 2019.

27. Duan CM, Zhang JR, Wan TF, Wang Y, Chen HS and Liu L: SRT2104 attenuates chronic unpredictable mild stress-induced depressive-like behaviors and imbalance between microglial M1 and M2 phenotypes in the mice. Behav Brain Res 378: 112296, 2020.

28. Lian N, Niu Q, Lei Y, Li X, Li Y and Song X: miR-221 is involved in depression by regulating Wnt $2 / \mathrm{CREB} / \mathrm{BDNF}$ axis in hippocampal neurons. Cell Cycle 17: 2745-2755, 2018. 
29. Iñiguez SD, Riggs LM, Nieto SJ, Dayrit G, Zamora NN, Shawhan KL, Cruz B and Warren BL: Social defeat stress induces a depression-like phenotype in adolescent male c57BL/6 mice. Stress 17: 247-255, 2014

30. Steru L, Chermat R, Thierry B and Simon P: The tail suspension test: A new method for screening antidepressants in mice. Psychopharmacology (Berl) 85: 367-370, 1985.

31. Livak KJ and Schmittgen TD: Analysis of relative gene expression data using real-time quantitative PCR and the 2(-Delta Delta C(T)) Method. Methods 25: 402-408, 2001.

32. Xie B, Lu C, Chen C, Zhou J and Deng Z: miR-135a alleviates silica-induced pulmonary fibrosis by targeting $\mathrm{NF}-\kappa \mathrm{B} /$ Inflammatory signaling pathway. Mediators Inflamm 2020: 1231243, 2020

33. Ferrúa CP, Giorgi R, da Rosa LC, do Amaral CC, Ghisleni GC Pinheiro RT and Nedel F: MicroRNAs expressed in depression and their associated pathways: A systematic review and a bioinformatics analysis. J Chem Neuroanat 100: 101650, 2019.

34. Allen L and Dwivedi Y: MicroRNA mediators of early life stress vulnerability to depression and suicidal behavior. Mol Psychiatry 25: 308-320, 2020.

35. Lou D, Wang J and Wang X: miR-124 ameliorates depressive-like behavior by targeting STAT3 to regulate microglial activation. Mol Cell Probes 48: 101470, 2019.

36. Xu B, Tao T, Wang Y, Fang F, Huang Y, Chen S, Zhu W and Chen M: hsa-miR-135a-1 inhibits prostate cancer cell growth and migration by targeting EGFR. Tumour Biol 37: 14141-14151, 2016.

37. Shi H, Ji Y, Zhang D, Liu Y and Fang P: miR-135a inhibits migration and invasion and regulates EMT-related marker genes by targeting KLF8 in lung cancer cells. Biochem Biophys Res Commun 465: 125-130, 2015.

38. Yan LH, Chen ZN, Li-Li, Chen J, Wei WE, Mo XW, Qin YZ, Lin Y and Chen JS: miR-135a promotes gastric cancer progression and resistance to oxaliplatin. Oncotarget 7: 70699-70714, 2016.

39. Zhou W, Li X, Liu F, Xiao Z, He M, Shen S and Liu S: miR-135a promotes growth and invasion of colorectal cancer via metastasis suppressor 1 in vitro. Acta Biochim Biophys Sin (Shanghai) 44: 838-846, 2012
40. Shi HZ, Wang DN, Xu F, Teng JH and Wang YL: miR-135a inhibits glioma cell proliferation and invasion by directly targeting FOXO1. Eur Rev Med Pharmacol Sci 22: 4215-4223, 2018.

41. Henn FA and Vollmayr B: Stress models of depression: Forming genetically vulnerable strains. Neurosci Biobehav Rev 29: 799-804, 2005

42. Wan YQ, Feng JG, Li M, Wang MZ, Liu L, Liu X, Duan XX, Zhang CX and Wang XB: Prefrontal cortex miR-29b-3p plays a key role in the antidepressant-like effect of ketamine in rats. Exp Mol Med 50: 1-14, 2018

43. Dey A and Hankey Giblin PA: Insights into macrophage heterogeneity and cytokineinduced neuroinflammation in major depressive disorder. Pharmaceuticals (Basel) 11: E64, 2018.

44. Iwata M, Ota KT and Duman RS: The inflammasome: Pathways linking psychological stress, depression, and systemic illnesses. Brain Behav Immun 31: 105-114, 2013.

45. Rush G, O'Donovan A, Nagle L, Conway C, McCrohan A, O'Farrelly C, Lucey JV and Malone KM: Alteration of immune markers in a group of melancholic depressed patients and their response to electroconvulsive therapy. J Affect Disord 205: 60-68, 2016.

46. Shen Z, Xu Y, Jiang X, Wang Z, Guo Y, Pan W and Hou J: Avicularin relieves depressive-like behaviors induced by chronic unpredictable mild stress in mice. Med Sci Monit 24: 1643-3750, 2018.

47. Du XJ and Lu JM: miR-135a represses oxidative stress and vascular inflammatory events via targeting toll-like receptor 4 in atherogenesis. J Cell Biochem 119: 6154-6161, 2018.

48. den Dekker WK, Cheng C, Pasterkamp G and Duckers HJ: Toll like receptor 4 in atherosclerosis and plaque destabilization. Atherosclerosis 209: 314-320, 2010.

49. Yang $L$ and Gao C: miR-590 inhibits endothelial cell apoptosis by inactivating the TLR4/NF- $\kappa \mathrm{B}$ pathway in atherosclerosis. Yonsei Med J 60: 298-307, 2019.

This work is licensed under a Creative Commons Attribution-NonCommercial-NoDerivatives 4.0 International (CC BY-NC-ND 4.0) License. 